\title{
Notas para una reflexión estratégica
}

\section{NOTES FOR AN STRATEGIC REFLECTION}

\author{
Rebeca Grynspan* \\ Bernardo Kliksberg**
}

\section{RESUMEN}

Notas para una reflexión estratégica es el resultado de un análisis de las condiciones de inequidad en las que actualmente se encuentra América Latina. En primera instancia, se caracteriza la región como la más inequitativa del planeta y se discuten las condiciones que plantean la necesidad de un nuevo debate para superar la inequidad. Se identifica el impacto sobre la cohesión social y la gobernabilidad democrática. Además de incidir en la pobreza, en las dificultades para enfrentarla, y en la cohesión social y la gobernabilidad, la desigualdad tiene efectos regresivos múltiples en la eficiencia de la economía, y las posibilidades de crecimiento sostenido. Por una parte estrecha las oportunidades de amplios sectores de poder desarrollar sus capacidades de inversión e innovación. Por otra crea condiciones para que sectores privilegiados agudicen las imperfecciones de mercado, y fomenten mecanismos de monopolización, protección de privilegios, capturas de rentas, en lugar de incentivos para la inversión y la innovación. En segunda instancia, se caracterizan las condiciones de desigualdad en América Latina al examinar indicadores en sectores específicos. A partir del reconocimiento de las disparidades en ingresos, participación en el mercado laboral, educación, salud, activos productivos, acceso al crédito, se configura el "accidentes de nacimiento", trampas cada vez mas rígidas de desigualdad, si es que no

\section{Abstract}

Notes for strategic thinking are the result of an analysis of the conditions of inequity in which Latin America is right now. In first instance, the region is characterized as the most unequal on the planet and discusses the conditions that posed the need for a new debate to overcome inequality. It identifies the impact on social cohesion and democratic governance. Besides influencing poverty, the difficulties to confront it, and social cohesion and governability, Inequality has multiple regressive effects on the efficiency of the economy, and the possibilities of sustained growth. On one hand reduces the opportunities for large segments to be able to develop their innovation and investment skills. On the other hand creates conditions for privileged sectors to intensify market imperfections, and promotes mechanisms for monopolization, protecting privileges, catches of income, instead of incentives for investment and innovation. In second instance, characterized the conditions of inequality in Latin America by examinating indicators in specific sectors. From the recognition of the disparities in income, participation in the labour market, education, health, productive assets, access to credit, "birth accident" is Configured, increasingly rigid traps of inequality, if public policies don't get actively involved providing opportunities to the disadvantaged. Finally proposed elements of response to the question, is inequity improvable?

\footnotetext{
Economista de la Universidad de Costa Rica. Socióloga de la Universidad Hebrea de Jerusalén. Obtuvo la Maestría en Economía en la Universidad de Sussex , Inglaterra. Directora de la PNUD, para América Latina y el Caribe. Costa Rica.

Ha obtenido cinco títulos Universitarios, entre ellos dos Doctorados, en economía y management. Ha asesorado a más de 30 países en alta gestión. Ha asesorado a diversos Presidentes y a numerosas organizaciones públicas de la sociedad civil y empresarial. Asesor de la ONU, BID, UNESCO, UNICEF, OIT, OEA, OPS y otros organismos internacionales. Ha sido galardonado por su trayectoria y contribuciones por diversos países. Se lo ha designado profesor Honorario, profesor Emérito. Doctor Honoris Causa de diversas Universidades del continente y huésped ilustre de diversas ciudades. Argentina.

Correo Electrónico: bernardo.kliksberg@undp.org

Articulo Tipo 2: de Reflexión. Según clasificación de Colciencias.

Fecha de Recepción: 20 de noviembre de $2007 \quad$ Fecha de Aprobación: 10 de diciembre de 2007.
} 
intervienen activamente políticas publicas que provean de oportunidades a los desfavorecidos. Por último se proponen elementos de respuesta a la pregunta ¿Es mejorable la inequidad? SE identifica un escenario propicio para el desarrollo de activas políticas proequidad que deben actuar estructuralmente sobre las dos caras de la inequidad, los "accidentes de nacimiento" y los “espacios de privilegio”. Está en esa dirección la nueva generación de políticas sociales en marcha en diversos países de la región que tiene entre sus ejes, la descentralización de los programas, la participación de las comunidades asistidas, el crecimiento de su capital social, el blindaje de los programas contra su instrumentación clientelar, la buena gerencia social, y el acceso a crédito, tecnologías, y oportunidades de mercados.

Palabras claves: Desarrollo Social, Cohesión Social, Inequidad, América Latina

\section{El NUEVo DEbate SObRe LaS DESIGUALDADES}

América Latina es según las mediciones de todas las fuentes tierra de profundas disparidades. Después de haberlo postergado durante largo tiempo, el nuevo debate público en la región viene integrando el tema cada vez más en al centro de la agenda nacional. Hay razones muy concretas para ello. Una es que el alto nivel de desigualdad es resentido fuertemente por sociedades civiles cada vez más participativas y articuladas. Entre el 80 y el $90 \%$ de los ciudadanos considera las tasas de inequidad existentes "injusta o muy injustas". Los ciudadanos advierten crecientemente que una explicación central de porque sociedades con una dotación privilegiada de recursos naturales y un inmenso potencial como las latinoamericanas presentan una pobreza persistente de gran magnitud, está en las intrincadas relaciones entre desigualdad y pobreza.

Desde las mediciones clásicas de Birdsall y Londoño (1997) demostrando que el aumento de desigualdad entre los 70 y los 90 , fue responsable del $50 \%$ del aumento de la pobreza, hasta las simulaciones econométricas recientes de CEPAL numerosas mediciones demuestran el impacto de la desigualdad sobre la alarmante pobreza latinoamericana.

El Banco Mundial (2005) estima que a bajos niveles de desigualdad un $1 \%$ de incremento en
It identifies a scenario conducive to the development of active proequity policies that should act structurally on the two sides of inequity, the "birth accidents" and the "spaces of privilege". It's in this direction the new generation of social policies on track in several countries of the region that has as one of its axes, decentralization of programs, aided community involvement, the growth of its social capital, the shielding of the programs against its client instrumentation, good social management, and access to credit, technology and market opportunities.

KeyWords: Develop Social, Social Cohesion, Inequity, Latin America

el Producto Bruto puede reducir la pobreza extrema en un 4\%. En cambio a altos niveles de desigualdad típicos de la mayor parte de los países de la región un aumento de un $1 \%$ en el Producto Bruto, tiene cero efectos en términos de reducción de pobreza extrema. En otros análisis (CEPAL, Programa Mundial de Alimentos, 2007) muestran como teniendo la región una producción de alimentos que supera tres veces las necesidades, un $16 \%$ de los niños presentan desnutrición crónica por las desigualdades de acceso.

CEPAL (2003) ha medido que los países con situaciones alimentarias más críticas podrían reducir a la mitad la población con hambre si disminuyeran moderadamente las desigualdades de acceso a los alimentos.

Por otra parte, la desigualdad afecta la cohesión social y la gobernabilidad democrática. Los ciudadanos saben que están viviendo en países con un gran potencial económico y donde núcleos reducidos de población logran niveles de vida comparables a algunas de las capitales de mayor bienestar del mundo. La convivencia de desigualdad y opulencia con pobreza relativa, y extrema, crean una situación de alta tensión social, y dañan severamente la cohesión social. El Consejo de Europa (2004) ha definido la cohesión social como "la capacidad de una sociedad para asegurar el bienestar de todos sus 
miembros, al minimizar las disparidades y evitar la polarización".

Los dos elementos básicos de la definición presentan serios déficits en la región. El bienestar de 205 millones de habitantes que viven por debajo de la línea de pobreza (38.5\% del total) no está asegurado, y la polarización es intensa. Esta última exacerba las insatisfacciones. No es lo mismo ser pobre en una sociedad parejamente pobre, a serlo en sociedades duales, donde coexisten niveles de vida como los de Ginebra, o Bruselas, con otros semejantes a los de países mucho menos desarrollados. La tensión que se genera es muy alta.

El clima de que esta dinámica no es "fair play" tiene un gran impacto en la desconfianza en las instituciones. Las grandes desigualdades son un destructor de capital social. Crean incredulidad, cinismo, falta de interés en la asociatividad, apatía, corroen el civismo. Los indicadores latinoamericanos de desconfianza hacia las instituciones básicas son muy altos como lo indican los resultados del Latinobarómetro (2006):

GRAFICO No. 1

Confianza en... America Latina 2006

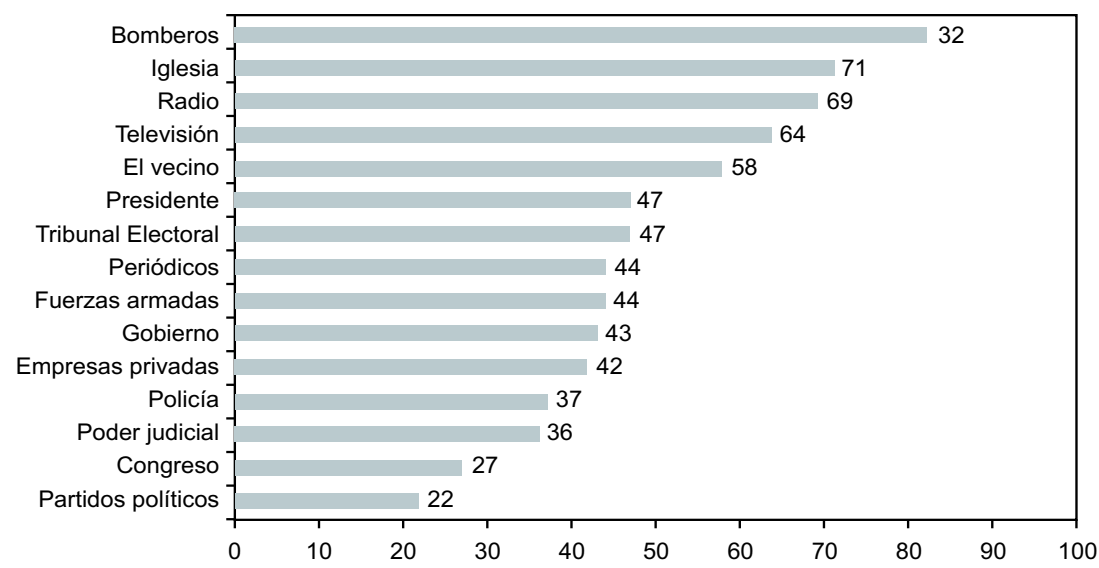

FUENTE: Latinobarómetro 2006. $n=20.234$

Como se observa encabezan la tabla de confianza instituciones claramente ajenas a la desigualdad, y que por el contrario la atenúan permanentemente, en cambio menos del $50 \%$ cree en las instituciones básicas, y menos del $25 \%$ en los partidos políticos, ambos incidentes en las oportunidades reales que tienen los ciudadanos. La falta de confianza, mina la legitimidad y reduce los márgenes de la gobernabilidad democrática. Es típica de las dificultades de legitimidad la respuesta que los latinoamericanos dan cuando se les consulta (Latinobarómetro 2006) sobre "para quien gobiernan los que gobiernan". El 69\% consideran que "son gobernados por unos cuantos grupos poderosos en sus propio beneficio". Hay un largo camino a recorrer para obtener la legitimidad plena necesaria para una eficiente gobernabilidad democrática. Parte del mismo pasa por crear condiciones de "juego limpio", reduciendo las inequidades.

Además de incidir en la pobreza, en las dificultades para enfrentarla, y en la cohesión social y la gobernabilidad, la desigualdad tiene efectos regresivos múltiples en la eficiencia de la economía, y las posibilidades de crecimiento sostenido.

Bourguignon y Walton (2007) abren una sugerente agenda de discusión al plantear que en realidad el tema "no es como alcanzar crecimiento con equidad, sino como entender las relaciones entre ambos". La inequidad genera por lo menos dos grandes dimensiones de ineficiencia. Por una parte estrecha las oportunidades de amplios sectores de poder desarrollar sus capacidades de inversión e innovación. Por otra crea condiciones para que sectores privilegiados agudicen las imperfecciones de mercado, y fomenten mecanismos de monopolización, protección de privilegios, capturas de rentas, en lugar de incentivos para la inversión y la innovación.

Esas dinámicas van a fortalecer asimetrías muy fuertes en la distribución del poder que a su vez incentivaran aun más la tendencia a crear o promover instituciones favorecedoras de los privilegios 
Los autores mencionados plantean que en realidad "es mala economía, y mala política" separar la desigualdad del crecimiento. Los daños de las altas inequidades son muy directos. En Suecia sólo mueren antes de cumplir un año de edad, el $0.5 \%$ de los niños, en Bolivia el 10\% entre las madres no educadas, 20 veces más. En la misma Bolivia la tasa desciende al $2 \%$ cuando se toma a madres educadas. La inequidad genera daños humanos, macroeconómicos, políticos, y sociales.

Pero además las disparidades graves son violatorias de valores éticos centrales del género humano. Independientemente de sus diferencias todas las creencias muestran actitudes prodignidad del ser humano, y de rechazo a condiciones que la ponen en cuestión. El Diálogo Mundial de las religiones sobre el desarrollo (1999) concluyó que: "todas las religiones ven a la extrema pobreza en el mundo actual como una ofensa a la humanidad y una ruptura de la confianza con la familia humana".

En teoría de los juegos se ha llegado en los últimos 10 años mediante experimentos, a rechazar la hipótesis convencional de que las personas sólo están interesadas en su bienestar individual. Según Fehr y Fischbacher (2003) un número significativo de gente muestra tendencias a dos tipos específicos de comportamiento: premiar y compensar a los que cooperan, y sancionar y castigar a los que sólo buscan su provecho. La equidad y el juego limpio les importan mucho.

También en estudios recientes sobre liderazgo cuando se dio a grupos la posibilidad de elegir líderes seleccionaron a aquellos que mostraban un carácter más solidario.

\section{LAS BRECHAS DE DESIGUALDAD LATINOAMERICANAS}

La desigualdad se halla presente en la región en casi todas las áreas de la economía. Normalmente los análisis se concentran en la distribución del ingreso, pero una visión mas integral debería incorporar otras dimensiones de fuerte impacto. Por otra parte las diversas desigualdades interaccionan a diario reforzándose mutuamente y generando "trampas de desigualdad" por un lado y "espacios de privilegio" por el otro que tienen un grado considerable de reproducción intergeneracional.

\subsection{INGRESOS}

Los últimos datos disponibles sobre distribución del ingreso (CEPAL 2006) son los que siguen:

\section{CUADRO 1}

América Latina (18 países): Estratificación de países según el coeficiente de GINI de la distribución del ingreso, alrededor de 1999 y 2005

\section{Nivel de desigualdad}

\begin{tabular}{|c|c|c|c|c|}
\hline $\begin{array}{l}\text { Muy Alto } \\
0,580-1\end{array}$ & $\begin{array}{l}\text { Brasil } \\
\text { Bolivia } \\
\text { Nicaragua }\end{array}$ & $\begin{array}{l}0,640 \\
0,586 \\
0,584\end{array}$ & $\begin{array}{l}\text { Bolivia (2.002) } \\
\text { Brasil } \\
\text { Honduras } \\
\text { Colombia }\end{array}$ & $\begin{array}{l}0,614 \\
0,613 \\
0,587 \\
0,584\end{array}$ \\
\hline $\begin{array}{l}\text { Alto } \\
0,520-0,579\end{array}$ & $\begin{array}{l}\text { Colombia } \\
\text { Paraguay } \\
\text { Honduras } \\
\text { Chile } \\
\text { Guatemala } \\
\text { Rep. Dominicana } \\
\text { Perú } \\
\text { Argentina } \\
\text { México } \\
\text { Ecuador }\end{array}$ & $\begin{array}{l}0,572 \\
0,565 \\
0,564 \\
0,560 \\
0,560 \\
0,554 \\
0,545 \\
0,539 \\
0,539 \\
0,521\end{array}$ & $\begin{array}{l}\text { Nicaragua (2.001) } \\
\text { Rep. Dominicana } \\
\text { Chile } \\
\text { Guatemala (2.002) } \\
\text { Paraguay } \\
\text { México } \\
\text { Argentinab }^{b}\end{array}$ & $\begin{array}{l}0,579 \\
0,569 \\
0,550 \\
0,542 \\
0,536 \\
0,528 \\
0,526\end{array}$ \\
\hline $\begin{array}{l}\text { Medio } \\
0,470-0,519\end{array}$ & $\begin{array}{l}\text { El Salvador } \\
\text { Panamáb } \\
\text { Venezuela R. B. } \\
\text { Costa Rica }\end{array}$ & $\begin{array}{l}0,518 \\
0,513 \\
0,498 \\
0,473\end{array}$ & $\begin{array}{l}\text { Ecuadorb }^{\text {b }} \\
\text { Perú } \\
\text { Panamáb } \\
\text { El Salvador } \\
\text { Venezuela R. B. } \\
\text { Costa Rica }\end{array}$ & $\begin{array}{l}0,513 \\
0,505 \\
0,500 \\
0,493 \\
0,490 \\
0,470\end{array}$ \\
\hline $\begin{array}{l}\text { Bajo } \\
0-0,469\end{array}$ & Uruguay ${ }^{\mathbf{b}}$ & 0,440 & Uruguay $^{\mathbf{b}}$ & 0,451 \\
\hline
\end{tabular}

Alrededor de 2.005

a Los valores límite del índice de Gini para cada categoría son los mismos que se emplearon en el capítulo I del Panorama social de América Latina 2004. Estos se determinaron empleando el algoritmo de estratificación estadística de las $\mathrm{k}$ medias, que busca generar estratos que sean homogéneos en su interior pero a la vez presenten la máxima variabilidad posible entre ellos. b Área urbana 
Puede observarse que predominan en la región desigualdades muy altas y altas. En el sexenio 1999-2005 ha habido cambios muy reducidos.
Los Gini latinoamericanos son muy superiores a los de los países desarrollados como puede observarse a continuación:

CUADRO 2

Indicadores de desigualdad para algunos países desarrollados

\begin{tabular}{lcc}
\hline & $\begin{array}{c}\text { Coeficiente } \\
\text { de Gini }\end{array}$ & $\begin{array}{c}\mathbf{1 0 \%} \text { más rico respecto } \\
\text { al 10\% más pobre }\end{array}$ \\
\hline Estados Unidos (2000) & 40,8 & 15,9 \\
Italia (2000) & 36,0 & 11,6 \\
Noruega (2000) & 25,0 & 6,1 \\
Suecia (2000) & 25,0 & 6,2 \\
Canadá (2000) & 32,6 & 9,4 \\
Finlandia (2000) & 26,9 & 5,6 \\
Dinamarca (1997) & 24,7 & 8,1 \\
España (2000) & 34,7 & 10,3 \\
\hline
\end{tabular}

FUENTE: Programa de las Naciones Unidas para el Desarrollo. Informe sobre Desarrollo Humano 2006.

El más alto Gini del grupo de países desarrollados es el de Estados Unidos 0.408 muy inferior al más bajo de la región, Uruguay 0.451 . El más alto de América Latina Brasil 0.614 más que duplica a los Noruega, Suecia, Finlandia y Dinamarca. El Gini latinoamericano es el más elevado de todas las regiones del mundo:

CUADRO 3

Coeficientes de Gini de la distribución de ingreso percápita por hogar:

Promedio simple por década por región

\begin{tabular}{ll}
\hline \multicolumn{1}{c}{ Región } & 1990s \\
\hline Niveles & \\
\hline América Latina y el Caribe & 52,2 \\
Asia & 41,2 \\
OCDE & 34,2 \\
Europa del Este & 32,8 \\
\hline
\end{tabular}

FUENTE: Cálculos basados en WIDER 2000, Smeeding y Grodner 2000, Székely, y aproximaciones para América Latina. Banco Mundial, Washington DC.

Según los datos del Banco Mundial (2004) el 10\% más rico de los latinoamericanos tiene el $48 \%$ del ingreso, mientras que el $10 \%$ más pobre sólo el $1.6 \%$. Reflejando las grandes distancias existentes entre los sectores sociales $3 / 4$ de los latinoamericanos tienen un per capita que es menor al per capita promedio, por lo que el $1 / 4$ restante tiene uno que es muy superior al promedio. Ello revela una vez más el carácter engañoso del per capita promedio como expresión del bienestar colectivo, en sociedades con altas disparidades.

\subsection{Mercado Laboral}

En la década de los 90 el $66 \%$ de todos los empleos creados lo fueron en el sector informal. El empleo evoluciono en ambos sectores como sigue: 
CUADRO 4

Distribución del empleo urbano en América Latina por

Segmento del mercado de trabajo y status laboral, 1990-1999

\begin{tabular}{l|c|c}
\hline \multirow{2}{*}{ Status Laboral } & \multicolumn{2}{|c}{ Proporción del empleo urbano } \\
\cline { 2 - 3 } & $\mathbf{1 9 9 0}$ & $\mathbf{1 9 9 9}$ \\
\hline Total de personas empleadas & 100.0 & 100.0 \\
Total del sector formal & 58.9 & 53.6 \\
Sector público & 16.0 & 12.9 \\
Sector privado & 44.4 & 41.3 \\
Empleados, profesionales independientes y & 3.8 & 4.3 \\
técnicos & & \\
Empleados & 40.6 & 36.9 \\
Profesionales y técnicos & 4.7 & 7.8 \\
No profesionales, trabajadores no técnicos & 35.9 & 29.1 \\
Total del sector informal & 41.0 & 46.3 \\
Empleo en microempresas & 14.7 & 15.5 \\
Empleo doméstico & 5.4 & 6.3 \\
Trabajadores autoempleados sin formación ${ }^{\text {a. }}$ : & 22.3 & 25.8 \\
En agricultura, selvicultura, caza y pesca & 2.2 & 3.0 \\
En Industria y construcción & 4.3 & 5.2 \\
En comercio y servicios & 15.8 & 17.7 \\
\hline
\end{tabular}

ancluye a empleadores y empleados en firmas de hasta cinco trabajadores.

FUENTE: CEPAL, de data de tabulaciones de encuestas de hogar conducidas en varios países, (2006).

A fines de la década los informales habían elevado su participación en el mercado de trabajo del $41 \%$ en 1990 al 46.3, y los puestos formales se habían reducido del 58.9 al 53.6. Ello implica que se había producido una fuerte degradación en la calidad de los trabajos, dado que la economía informal implica menor productividad, falta de protección social, $\mathrm{y}$ menores ingresos. En 1990 el ingreso promedio de los empleados en el sector formal era $60 \%$ más alto que el de los trabajadores informales. En el 2000 esa relación había aumentando, al 72\% (Machinea \& Kacef 2007). También las desigualdades son agudas en el campo del desempleo. Durante los 90 el desempleo abierto creció del 4.6 al 8.6\%. El desempleo promedio entre el $40 \%$ mas pobre de la población fue considerablemente más alto que las tasas promedio.

\subsection{Educación}

Han sido notables los progresos en matriculación en primaria y reducción del analfabetismo, pero las desigualdades sociales los han minado. Independientemente del trabajo de la escuela, dichas disparidades impactan a los sectores más pobres, generando altas tasas de deserción. Cerca de la mitad de los que comienzan la primaria no la terminan. Entre las causas principales: el trabajo infantil (20 millones de niños menores de 14 años trabajan), la desarticulación familiar, la desnutrición, el hacinamiento. Las diferencias en grados de escolaridad son muy acentuadas. Según los estimados de CEPAL el mínimo de escolaridad necesaria para obtener un empleo decente son 12 años. El $75 \%$ de los jóvenes urbanos vienen de hogares en donde los padres han tenido menos de 10 años de escolaridad. En promedio el $45 \%$ de ellos no llegan a los 12 años. En las zonas rurales, es peor es el $80 \%$ (SAINZ 2006). Sólo el $30 \%$ de los hijos de padres que no terminaron primaria, termina la secundaria.

Por otra parte refuerza las diferencias el hecho de que el sector de alta escolaridad tiene a su vez una educación de mucha mejor calidad que el de baja escolaridad. Según los datos de DELICH (2002) el estudiante promedio de la escuela publica solo ve el $50 \%$ del curriculum, el de escuela privada el 100\%. En los tests el $40 \%$ de los niños de cuarto y quinto grado de bajo nivel socioeconómico, no entienden lo que leen.

Los niveles de educación diferenciales van a incidir en múltiples aspectos. Uno muy importante son las brechas salariales. Según los estimados de CEPAL (2006) la educación es la causante del $38 \%$ de dichas brechas y su importancia viene aumentando. 
Investigaciones comparadas indican que se paga muy caro la inequidad en educación. Un estudio reciente en Estados Unidos determino que una persona que no completa la secundaria vive 9.2 años menos que aquella que la termina. El estado de salud de un desertor del secundario a los 45 anos, es peor que el de un graduado del secundario a los 65 años.

\subsection{SALUd}

Hay progresos relevantes en los indicadores promedio de la región en salud. Sin embargo cuando se desagregan aparecen importantes brechas entre países y al interior de los mismos. Dada la trascendencia definitoria de la salud, base estratégica para el desempeño esas brechas van a repercutir en todos los ordenes.

Las cifras de mortalidad infantil de Bolivia multiplican casi 9 veces las de Chile. Mueren 70 niños de cada mil antes de cumplir 5 años de edad, frente a 8 en Chile. Cuando se abren los promedios la mortalidad infantil del 20\% más pobre de Bolivia es cinco veces la del $20 \%$ más rico. La misma proporción se repite en Perú. En Brasil la diferencia es 3 a 1 .

Las cifras de desnutrición crónica alcanzan el $16 \%$ promedio para toda la región. Suben al $38,5 \%$ en el $10 \%$ más pobre de Ecuador, y llegan en la población indígena de dicho país al $58 \%$. En Perú son del $49.6 \%$ en el $10 \%$ más pobre, y del $47 \%$ en la población indígena.

Las posibilidades de que una madre muera al dar a luz son 1 entre 7300 partos en el mundo desarrollado. En América Latina con progresos son 1 entre 160. Cerca de una quinta parte de las madres no tienen asistencia, durante el embarazo.
En el 20\% más pobre de Bolivia y de Perú la cifra se triplica.

La desigualdad en factores de riesgo claves en salud como agua potable y saneamiento es muy pronunciada. Hay en la región 50 millones de personas que carecen de agua en buenas condiciones. Están concentradas en los sectores de menores ingresos. Mientras que en Perú en el $20 \%$ más rico el $100 \%$ tenía agua potable, en el $20 \%$ más pobre era sólo el 25\%. En Colombia las proporciones eran $100 \%$ vs. $41 \%$. En Bolivia el acceso era en la población no indígena del $80 \%$ y en la indígena del $49 \%$.

Mientras que para el 20\% más rico de la región el costo del agua es un porcentaje muy reducido de sus gastos, para los pobres que se ven obligados a comprarla es mucho mayor. El gasto en agua en el $10 \%$ más pobre triplica al del $10 \%$ más rico en los centros urbanos de Ecuador y Brasil. En el 20\% más pobre de El Salvador, Jamaica y Nicaragua representa el $10 \%$ de los gastos.

En materia de saneamiento 120 millones de latinoamericanos carecen de una infraestructura adecuada. En Brasil la cobertura no llegaba al $50 \%$ en aquellos hogares cuyo ingreso es un salario mínimo.

\subsection{Activos Productivos}

La posibilidad de acceder a tierra productiva es muy desigual en la región. Los coeficientes Gini respectivos superan ampliamente los de todas las otras regiones, y son mucho mayores aun a los deficientes Gini de distribución del ingreso antes referido.

El cuadro siguiente ilustra al respecto:

CUADRO 5

Coeficientes Gini de distribución de la propiedad de la tierra

\begin{tabular}{cccc}
\hline Región & D\&O (i) & UNDP (ii) \\
\hline América Latina & 0,81 & 0,74 \\
Medio Oriente y África del Norte & 0,67 & 0,56 \\
Norteamérica & 0,64 & 0,51 \\
África Sub-Sahara & 0,61 & 0,52 \\
Europa Occidental & 0,57 & 0,56 \\
\hline
\end{tabular}

Nota: Columna (i) muestra los promedios del periodo 1950-1994; Columna (ii) muestra los valores alrededor del 1981. 


\subsection{Acceso Al Crédito}

El acceso al crédito es muy asimétrico en la región y ello va a incidir fuertemente en las posibilidades de desarrollar emprendimientos productivos. El sistema financiero tiende a presentar una alta concentración, y privilegiar el crédito a las empresas de mayor dimensión. Los niveles mismos de bancarización son limitados en amplios sectores. Así en Ciudad México sólo el $25 \%$ de los hogares hacen uso de los servicios bancarios. Por otra parte con avances considerables el microfinanciamiento tiene una cobertura reducida, y es mas bajo que en otras regiones. Mientras en Bangladesh, Indonesia, y Sri Lanka llega al 8\% de la población en los países latinoamericanos va de proporciones casi ínfimas en las economías mayores como Brasil, México y Argentina, hasta un 4\% de la población en el país con tasa más alta que es Bolivia, como puede apreciarse en el siguiente gráfico:

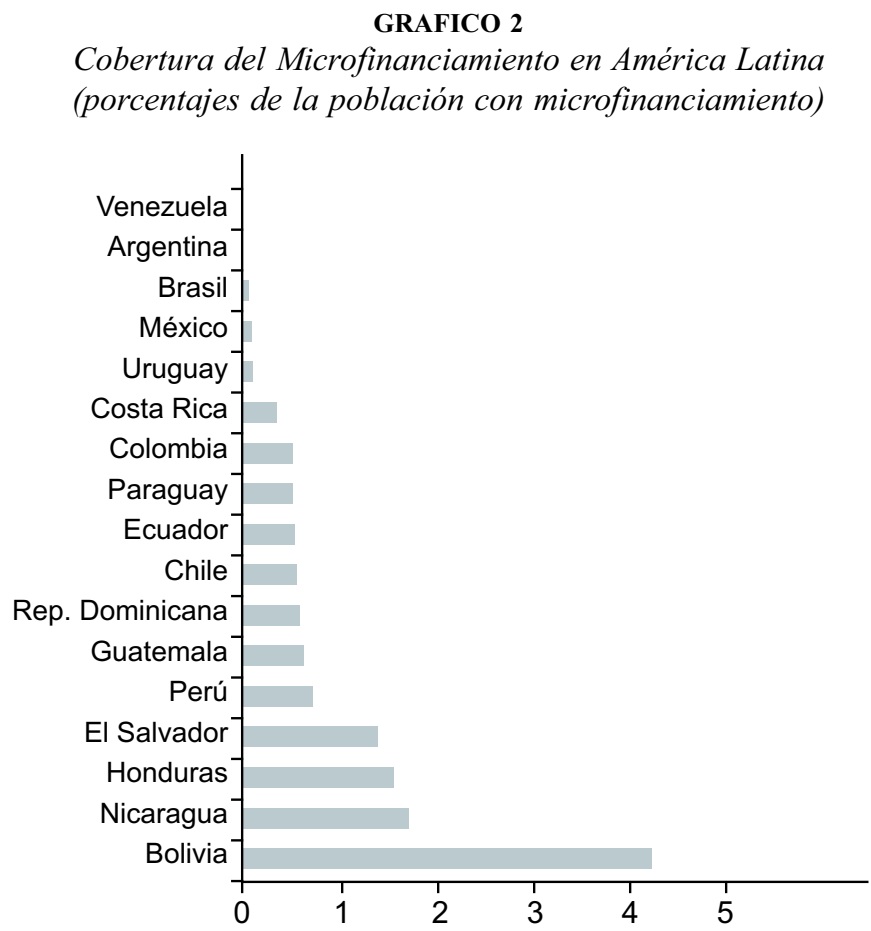

Clientes de préstamos como porcentaje de la población total

FUENTE: Honohan P. (2004)

\subsection{Las Trampas de Desigualdad en Acción}

Las disparidades en ingresos, participación en el mercado laboral, educación, salud, activos productivos, acceso al crédito, y otras añadibles se refuerzan unas a otras.

Configuran en su conjunto una situación que puede denominarse el "accidente de nacimiento". Los niños no tienen ninguna posibilidad de influir sobre en que país, y región del mismo van a nacer, cual será el nivel de educación de sus padres, cual su posicionamiento socioeconómico, y cual será su nivel nutricional y de salud inicial. Todo ello será sin embargo determinante en sus vidas.

En un estudio en Brasil (Universidad de Sao Pablo 1996) se verifico que la incidencia de peso menor al que correspondería según la edad era del 19.9\% cuando las madres tenían menos de 3 años de educación formal, y seis veces menor $3.35 \%$ cuando tenían 11 o más años de educación.

Paxson y Chady (2005) hallaron en Ecuador en niños de 3 a 6 años, que el desarrollo cognitivo tenia una correlación robusta con factores como 
los ingresos del hogar, y el nivel educacional de las madres.

Las interrelaciones funcionan permanentemente convirtiendo los "accidentes de nacimiento" en trampas cada vez mas rígidas de desigualdad, si es que no intervienen activamente políticas publicas que provean de oportunidades a los desfavorecidos.

Las trampas pueden tomar todo tipo de formas. Así por ejemplo los sectores de menores ingresos tienden a postergar sus necesidades de atención en salud de modo sistemático.

En Paraguay (OPS, 2003) se halló que el 51\% de la población que declaro haber tenido enfermedades o accidentes en los tres meses anteriores no consulto sin embargo ningún establecimiento medico.

Diversos estudios encontraron fuertes correlaciones entre logros educativos y esperanza de vida. En Chile (Vega \& Otros, 2003) hallaron que la diferencia entre los grupos con mayor y menor nivel educativo era de 4.7 años. Messias (2003) encontró en el Brasil estudiando comparativamente los diversos Estados que un $10 \%$ mas de analfabetismo significa 2.2 anos menos de esperanza de vida.

\section{3. ¿LA EQUIDAD ES MEJORABLE?}

En las últimas décadas se ha tendido con frecuencia en la región a colocar la posibilidad de mejorar la equidad en el rango de un subproducto más de la obtención de un crecimiento elevado. El crecimiento de por si "derramaría" equidad. Los intentos de apresurar ese camino podrían ser incluso nocivos para el crecimiento y retardarlo.

Esta línea de pensamiento ha chocado con la experiencia histórica concreta. Ella esta indicando que la situación es muy disímil. La falta de equidad es un obstáculo formidable para un crecimiento sostenido. Genera entre otros aspectos reducciones importantes en el tamaño de los mercados internos, bajas tasas de ahorro nacional, déficit de envergadura en la calidad educativa de la población factor central para la competitividad en una economía mundial cada vez mas "conocimiento intensiva", destrucción de capital social, un costo enorme en términos de potencial desaprovechado de la población, fracturas considerables en la cohesión social, y conduce a climas conflictivos, y baja gobernabilidad. Ello a su vez obstruye el fortalecimiento de las instituciones, y retrae la atracción de inversiones.

Por parte las altas disparidades propias de la región crean restricciones múltiples a la conversión del crecimiento en reducción efectiva de la pobreza. Generan una inelasticidad de los niveles de pobreza ante el crecimiento, porque están operando activamente las "trampas de desigualdad" que impiden que los atrapados en ellas reciban sus efectos.

El mejoramiento de la equidad es una clave decisiva para obtener en la región muchos mejores niveles de eficiencia, construir un crecimiento sostenible en el tiempo, recuperar la confianza, y afianzar la gobernabilidad democrática.

Frente al peso de los hechos, las reservas de algunos sectores respecto a la necesidad de mejorar de modo efectivo la equidad, se han concentrado en la viabilidad de lograrlo. Seria una meta valida como tal, pero no realista.

La experiencia internacional no da mayores avales a este razonamiento. Indica claramente que es un camino complejo, y que requiere políticas innovativas, coherentes, y con visión de mediano y largo plazo, pero que se pueden obtener progresos considerables.

Experiencias económicas altamente exitosas recientes han tenido como uno de sus ejes la apuesta por vigorosas políticas creadoras de oportunidades para todos. Países como los nórdicos Noruega, Finlandia, Suecia, Dinamarca, que se hallan entre los primeros lugares de los rankings de desarrollo humano, desarrollo sostenible, progreso tecnológico y competitividad, y entre otros Corea, y Taiwán han realizado inversiones iniciales de gran magnitud y sostenidas en el tiempo en universalizar educación de buena calidad, y en caso de estos últimos en posibilitar el acceso a la propiedad de la tierra. Japón adoptó como uno de los pilares de su reconstrucción después de la guerra el establecimiento de un sistema de salud universal. Informes comparativos detallados sobre las economías más exitosas como "Macroeconomía y salud" (Who, 2002) dirigido 
por Sachs, señalan que es común a la gran mayoría de ellas que no dejaron la inversión en salud para después que hubiera un crecimiento importante, sino que la consideraron una condición previa imprescindible y dedicaron importantes recursos a la dotación de infraestructuras de agua y saneamiento, la reducción de la mortalidad infantil y materna, y la creación de extendidas coberturas de salud.

Este orden de políticas generadoras netas de equidad contribuyeron decisivamente a formar una población de alta calidad que fue y es fundamental en la inserción competitiva exitosa que han logrado esas economías.

En América Latina hay actualmente un escenario muy propicio para el desarrollo de activas políticas proequidad. Hay una "ventana de oportunidad" muy significativa al respecto. La constituyen condiciones favorables estratégicas como entre otras el buen desempeño macroeconómico de los últimos años, las ganancias democráticas, y el firme apoyo que estas políticas tienen en las expectativas de la población.

Existen asimismo amplias posibilidades de convocar a pactos nacionales sobre estas políticas que puedan transformarlas en verdaderas políticas de Estado.

Se tendría que actuar estructuralmente sobre las dos caras de la inequidad, los "accidentes de nacimiento" y los "espacios de privilegio". En el primer caso, las metas son expandir oportunidades para los sectores postergados, y facilitar y promover la inclusión productiva. Los resultados iniciales de nuevas políticas y programas que apuntan hacia la pobreza pero con una visión inclusiva y no solamente atenuadora son promisorios. Esta en esa dirección la nueva generación de políticas sociales en marcha en diversos países de la región que tiene entre sus ejes, la descentralización de los programas, la participación de las comunidades asistidas, el crecimiento de su capital social, el blindaje de los programas contra su instrumentación clientelar, la buena gerencia social, y el acceso a crédito, tecnologías, y oportunidades de mercados. También se halla en la misma vía la recomposición de la inversión en educación y salud que se esta produciendo en diversos países. Hay un amplísimo camino a recorrer en estas áreas, en dirección a políticas universalizadoras. También en la conformación de un mercado financiero que facilite el acceso a crédito a las PYMES y las microempresas, y en servicios tecnológicos, y de mercado que las apoyen de modo sistemático. El espectro de políticas necesarias es muy vasto y va desde reforzar los programas de intervención temprana para mejorar la situación de los niños pobres, hasta la inversión en escala en agua y saneamiento sostenible. Un eje fundamental será el de articular solidamente las políticas económicas y las sociales. Por otra parte para recortar los "espacios de privilegio" se requerirán políticas que actúen a favor de la democratización de los mercados. También será necesario profundizar la discusión sobre un nuevo pacto fiscal que de bases mas sólidas a las inversiones sociales que se requieren, que establezca una distribución de fuentes impositivas mas equitativa, y reduzca al mínimo la evasión. El argumento de que un aumento de la presión fiscal generara necesariamente un retroceso económico, parece ser cada vez más débil a la luz de las experiencias comparadas. Las economías más desarrolladas muestran como un rasgo común un significativo aumento de dicha presión en las ultimas tres décadas como lo muestran las cifras siguientes:

\section{GRAFICO 3}

Incremento del costo del Gobierno. La proporción del producto interno bruto destinado a losImpuestos ha aumentado desde 1975 en la mayoría de los países

\section{INGRESO FISCAL COMO PORCENTAJE DEL PIB}

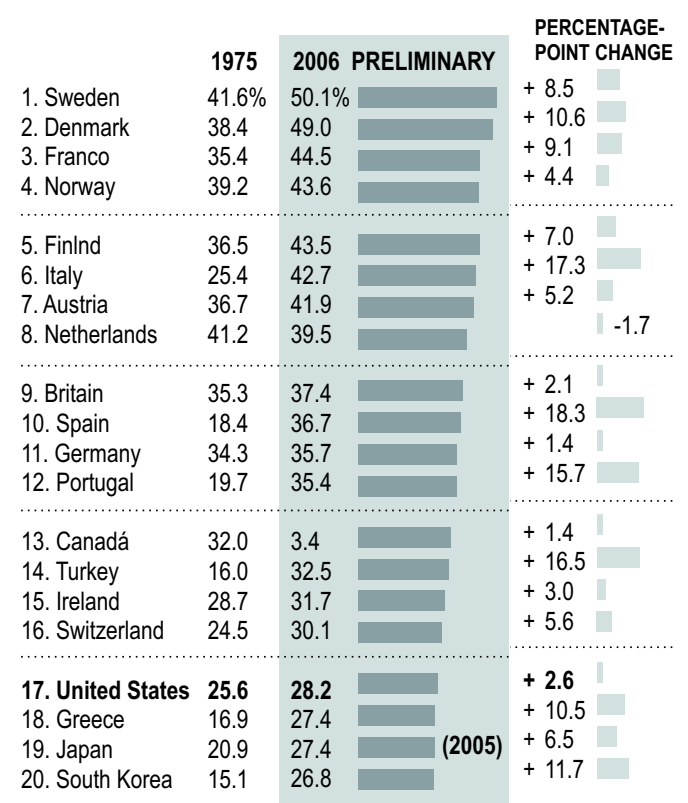

FUENTE: Organización para la cooperación económica y el desarrollo. October 18, 2007. The New York Times 
Como se advierte la presión fiscal ha crecido considerablemente en los países desarrollados entre 1975 y el 2005 . Ha pasado de un promedio de $30 \%$ a un $36 \%$ en ese periodo, incluido Estados Unidos donde aumento en un $2.6 \%$. Heady (2007) Director de Política fiscal de la OECD resalta el caso de Suecia, "que tiene la más alta presión fiscal de toda la OECD superior al $50 \%$, y $\sin$ embargo es uno de los países de la ECD de mejor desempeño económico en los últimos 20 años". La presión fiscal en los países de la OCDE duplica virtualmente la latinoamericana, a pesar de tener déficit sociales mucho menores.
Por otra parte esta basada en muchos de ellos en sistemas mucho más progresivos que los de la región. Una América Latina que esta en pleno proceso de cambios positivos en la macroeconomía, la estabilidad, y la democratización, debe poner en primera línea de sus prioridades mejorar la equidad. No es un tema más, hacerlo será definidor para la configuración de economías y sociedades de real solidez, y calidad. Por otra parte no parece ser una opción. Es una demanda planteada en forma cada vez más aguda y exigente por la ciudadanía.

\section{REFERENCIAS BibliográficaS}

Banco Mundial (2000). Desigualdad en América Latina y el Caribe. ¿Ruptura con la historia?, Washington DC: Banco Mundial

Birdsall, N. \& Londoño, J.L. (1997). Assets inequality matters. An assessment of the World Bank's approach to poverty reduction. American Economic Review. Mayo.

Bourguignon, F. \& Walton, M. (2007). Is greater equity necessary for higher long-term growth in Latin America?. En French-Davis R. \& Machinea J.L. (Eds.)/(Comps.), Economic Growth with Equity. Challenges for Latin America. Santiago de Chile: ECLAC, Palgrave Macmillan.

Comisión Económica para América Latina y el Caribe - CEPAL - (2003). Panorama Social de América Latina 2002-2003. Santiago de Chile: NACIONES UNIDAS \& CEPAL.

Comisión Económica para América Latina y el Caribe - CEPAL - (2006). Panorama Social de América Latina. Santiago de Chile: NACIONES UNIDAS \& CEPAL

Corporación Latinobarómeto (2006). Informe Latinobarómetro. Recuperado el año 2006, de www.latinobarometro.org

Deininger and Olinto (2002) and UNDP (1993). En, Banco Mundial (Ed.). Desigualdad en América Latina y el Caribe.

Delich, V. (2002). Developing countries and the WTO dispute settlement system. En: Handbook, A., Bernard, H., Aaditya, M. \& Philip English (eds.). Development, Trade and the WTO. Washington D.C.: World Bank.

Fehr, E. \& Fischbacher, U. (2003). The nature of human altruism. Nature, 425 October.

Heady, C. (2007). En Johnston, David Vay. Taxes in developed nations reach $36 \%$ of gross domestic product. The New York Times, October 18.

Honohan, P. (2004). Financial Developmetn, Grwoth, and Poverty: how Close are the links? Policy Research Working Paper, Series 3203.

Machinea, J.L. y Osvaldo L.K. (2007). Growth and Equity: in search of the "empty box". En R. French-Davis \& J.L, Machinea (Eds.)/(comp). Economic Growth with equity. Challenges for Latin America. Santiago de Chile: ECLAC, Palgrave Macmillan.

Messias, E. (2003). Income inequality, illiteracy rate, and life expectancy in Brazil. American Journal of Public Health, 93 (8) August.

Organización Panamericana de la Salud - ASDI - (2003). Exclusión en salud en países de América Latina y del Caribe. Washington DC: ASDI

Paxson, Ch.H. \& N. Schady (2005). Cognitive development among young children in Ecuador: the role of wealth, health and parenting. World Bank Policy Research. Working Paper Series, No.3605.

Sainz, P. (2006). Equity in Latin America since the 1990s. Economic and Social Affair. DESA Working paper No.22.

Programa de las Naciones Unidas para el Desarrollo (2006). Informe sobre Desarrollo Humano. Recuperado en el año 2007, de: http://www.undp.org/spanish/publicaciones/informeanual2006/ desarrollo-humano.shtml 
Programa Mundial de Alimentos, CEPAL (2007). El costo del hambre. Centro América y República Dominicana. Recuperado en el año 2007, de: http://www.cepal.org/publicaciones/xml/3/28923/ DP CostoHambre.pdf

The New York Times (2007). Tabla sobre el ingreso fiscal como porcentaje del PIB de la Organización para la cooperación económica y el desarrollo. The New York Times, Publicado el 18 de Octubre.

The World Bank (2005). World development report 2006. Equity and development. Washington DC.: The World Bank

The World Health Organization (2002). Macroeconomic and health: investing in health for economic development. Geneve.

Universidad de Sao Pablo (1996). Estudios epidemiológicos en nutrición. Escuela de Salud Pública.

Vega Jeannette, y otros (2003). Chile: diferenciales socioeconómicos y mortalidad en una nación de ingreso medio. En "Desafio a la falta de equidad en la salud". Washington DC: OPS, Fundación Rockefeller. 